\title{
Mathematics and Conceptual Analysis
}

\author{
Antony Eagle (antony .eagle@philosophy.ox.ac.uk) \\ Oxford University
}

\begin{abstract}
Gödel argued that intuition has an important role to play in mathematical epistemology, and despite the infamy of his own position, this opinion still has much to recommend it. Intuitions and folk platitudes play a central role in philosophical enquiry too, and have recently been elevated to a central position in one project for understanding philosophical methodology: the so-called 'Canberra plan'. This philosophical role for intuitions suggests an analogous epistemology for some fundamental parts of mathematics, which casts a number of themes in recent philosophy of mathematics (concerning a priority and fictionalism, for example) in revealing new light.
\end{abstract}




\section{Introduction}

Gödel's views on the epistemology of mathematics are notorious: notoriously difficult to interpret and - no matter how sympathetically interpreted - notoriously implausible. Nevertheless, there is something to the observations he makes about the importance of intuition in mathematical epistemology; despite the overall implausibility of Gödel's quasi-perceptual view of intuition, his insistence on the importance of intuitions has much to recommend it. In the present paper, I wish to sketch a kind of intuition-based epistemology for foundational mathematics that respects the force behind Gödel's observations, while retreating from the details of his perceptual account of intuition. Though Gödel would find the resulting picture uncongenial, I believe the picture proposed here is of considerable interest, not least because of the light it sheds on the relationship between the traditionally a priori disciplines of mathematics and philosophy.

After some brief remarks on Gödel's views on intuition in mathematics (§2), I develop the thought that the best way to understand the significance of intuitions is in their role as data for conceptual analysis. In §3, I describe one recent influential account of conceptual analysis, given by the so-called 'Canberra Plan', that gives a central role to intuitions. In §4, I apply the Canberra plan to the role of intuitions in mathematics, and give an account of mathematics as a kind of conceptual analysis. This account sheds quite striking new light on some traditional features of mathematics. In §5 I try to resolve some problems that arise because of the generality of mathematical concepts. In $\S 6$ I try to answer a fundamental objection concerning the existential commitments of mathematical axioms; surprisingly, this connects the present account quite intimately with recent debates over fictionalism in mathematics.

Two disclaimers before beginning. Firstly, I am not proposing a descriptive account of how mathematical practice actually proceeds (though the picture does fit some historical developments in mathematics). It is rather a rational reconstruction of how epistemic access to some mathematical truths can be grounded.

Secondly, the paper deals only with a small part of mathematics that I term 'foundational'. This term is intended to refer to basic arithmetic, set theory, and parts of geometry. The term 'foundational' is appropriate: these branches of mathematics are in practice the foundation for the rest of our mathematical development, as it is our grasp and facility with the concepts of these areas 
that largely grounds our competence with higher mathematics. The view I propose regards this conceptual priority of arithmetic and set theory as good grounds for their special epistemic status. And, of course, set theory is widely regarded as foundational in another sense: many still believe that a reduction of all mathematicalia to set theoretic constructions can be successfully and plausibly undertaken.

I well realise that current fashion in the philosophy of mathematics normally dictates more attention to higher mathematics and to mathematical practice than appears in this paper. I completely agree with the thought that an over-emphasis on arithmetic and set theory did for some time hamper the progress of philosophy in understanding mathematics. Yet just because our gaze has now widened doesn't mean that the epistemological and ontological problems of arithmetic and set theory have been solved: far from it. Furthermore, there is good reason to think that whatever philosophical story we tell about foundational mathematics should be quite different to the story we will tell about higher mathematics. Phenomenologically, foundational mathematics is distinctive: one really does have intuitions about how collections and numbers should behave that are quite independent of one's mathematical sophistication. In that sense I think it would be a failing of philosophy of mathematics if our account made elementary arithmetic just as easy (or just as hard) to know as higher analysis. The hope of the present paper is that a reasonably plausible account of some parts of mathematics can be given, and that higher mathematics may be 'bootstrapped' in some yet to be determined fashion from these foundational parts. I make no attempt to describe that bootstrapping process here. For now it must suffice to say that it will look very dissimilar to the account I propose here - the extension of the number concept to encompass complex numbers doesn't seem, for example, to involve platitudes at all, but rather to involve a subtle interplay between increased generality and radical conceptual innovation.

\section{Gödel on Mathematical Intuition}

A caricature of Gödel's own views is quite familiar to philosophers of mathematics. This caricature view is that knowledge of mathematical facts is immediately given by a quasi-perceptual faculty of mathematical intuition, giving direct access to mathematical objects and their properties. Yet Gödel's actual views are not nearly so simplistic or straightforward, and there is little evidence that he was deeply committed to any such quasi-perceptual view. ${ }^{1}$ Since I'm not anachronistically arguing that Gödel himself held the view I'm going to be arguing for, I won't take too much time over the details of his remarks. Rather I will draw out one suggestive thread from what he says, especially in his later work, that will serve to motivate the view I defend. 
Gödel's position on mathematical entities was fundamentally a realist one. He claims that our mathematical beliefs

may represent an aspect of objective reality, but, as opposed to the sensations, their presence in us may be due to another kind of relationship between ourselves and reality. (Gödel, 1964, 484)

This 'other kind of relationship' involves sensitivity to mathematical truth in virtue of some non-perceptual epistemic capacity, which Gödel dubbed intuition.

There is considerable evidence that Gödel sometimes regarded intuition as giving us access to the truth of certain privileged mathematical propositions: the axioms. For Gödel, one has an intuition that $\phi$ just in case $\phi$, when entertained, is undeniable: 'the axioms force themselves upon us as being true' (Gödel, 1964, 484). This is presumably in virtue of the concepts involved in the proposition $\phi$, and our ability to grasp how different concepts that we possess relate to one another. ${ }^{2}$ Gödel thus emphasises that, for mathematical knowledge, the concepts in question must be sufficiently clearly articulated for us to be able to respond in this immediate way to propositions involving them:

mathematical concepts must be sufficiently clear for us to be able to recognize their soundness and the truth of the axioms concerning them.... (Gödel, 1964, 474)

Indeed, getting a clearly articulated grasp of the concepts is not merely prerequisite for mathematical knowledge: it is the whole story. When considering possible extensions to ZFC set theory, he remarks

set theory ... can be supplemented without arbitrariness by new axioms which only unfold the content of the concept of set. (Gödel, 1964, 477)

And further:

The mere psychological fact of the existence of an intuition which is sufficiently clear to produce the axioms of set theory and open series of extensions of them suffices to give meaning to the question of the truth or falsity of propositions like Cantor's continuum hypothesis. (Gödel, 1964, 484)

These quotations suggest very neatly the idea that merely getting clear on what is and is not part of the concept of set will be able to give us access to mathematical truths in a reliable ('non-arbitrary') way.

All this suggests that when we have a mathematical intuition, this involves a certain axiomatic claim appearing to us as undeniably true, in virtue of the concepts involved in that claim. The claim is certain to the extent that the concepts involved are clearly articulated, which makes it a pressing task to explicate just what the content of these concepts is. The claim is intuitive to the extent that the truth of the claim is guaranteed by the relations between the concepts: the axiom merely 'unfolds' the consequences of the content of 
the concepts. In this way, the intuitive truth of ' $2+2=4$ ' might be supposed exactly analogous to 'nothing is both round and square' (when that is read as a claim about physical shapes, not geometric objects). ${ }^{3}$

That mathematical claims are analytic is part of Gödel's view, and it is a position with a long and distinguished heritage. But the other part of Gödel's view, that the truth of the axioms is supposed to be secured by understanding the content and soundness of the concepts involved, is more unusual at least in the foundations of mathematics. To philosophers, it should look awfully familiar, as it appears to involve good old-fashioned conceptual analysis. Trenchant criticism of old-fashioned conceptual analysis, by Quine and others, probably explains why this aspect of Gödel's thought wasn't pursued. But there is a contemporary proposal that reanimates the prospects of conceptual analysis, and may be able to revive the prospects for intuition playing a role in the foundations of the mathematics.

\section{The 'Canberra Plan' and Conceptual Analysis}

The proposal concerning conceptual analysis I have in mind has become known as the Canberra Plan; its most prominent contemporary defender is Jackson (1998), but its roots go back at least to Lewis (1970). ${ }^{4}$ I begin by looking at his view of supervenience and inter-theoretical reduction.

Deciding whether, and how, theories about different topics can be unified is a significant question in the metaphysics of science. By 'theory' I just mean (the set of) models for a discourse concerning some subject matter (van Fraassen, 1989, ch. 9). ${ }^{5}$ There are of course theories of physics and chemistry; but there are are theories of minds, colours, and meanings too, because there are discourses about those topics that are both systematic and truth-apt. Jackson claims that we may unify these disparate theories by locating the concepts of one theory in another theory:

The one and only way of having a place in an account told in some

preferred terms is by being entailed by that account. (Jackson, 1998, 5)

This semantic entailment thesis corresponds to a reductionist supervenience thesis. Call a model of $T$ minimal just in case it includes only properties mentioned in $T$. A theory $T_{1}$ supervenes on $T_{2}$ iff every minimal model of $T_{2}$ is a model of $T_{1} \cdot{ }^{6}$

It turns out that, according to Jackson,

conceptual analysis is the very business of addressing when and whether a story told in one vocabulary is made true by one told in some allegedly more fundamental vocabulary. (Jackson, 1998, 28)

We locate the concepts of one theory in another theory if we can give an analysis of the former concepts in the terms of the latter. So the epistemic 
question of how to tell when one theory supervenes on another can only be conclusively answered by analysing one theory in terms of the concepts and properties provided by the other. ${ }^{7}$ These analyses need not be 'analytic', because inter-theoretic reductions needn't be purely definitional. That is, there might be multiple mappings from one theory to another; or there may be no perfect mapping that preserves the entirety of $T_{1}$, and hence there may be an element of flexibility in locating the concepts of $T_{1}$ in $T_{2}$. This sense of 'analysis' is more like what Carnap termed 'explication': the reduction of one property (corresponding to a concept) to other properties that best match the original property.

Jackson suggests that the success of a conceptual analysis can be determined by examining possible cases. So $\mathcal{R}$ is a successful analysis of $\mathcal{K}$ iff possible cases of something's being $\mathcal{K}$ are all and only cases of that something's being $\mathcal{R}$. Whether this can be applied depends on our mastery of the concepts in question, and particularly on our ability to correctly use the words that express those concepts. Jackson thinks that intuitions about how to classify possible situations are a reliable guide to the correct classification of those cases, and hence to the adequacy of conceptual analyses involving those concepts:

consulting intuitions about possible cases is simply part of the overall business of elucidating concepts by determining how subjects classify possibilities. (Jackson, 1998, 33)

Of course, the mere fact that people instinctively classify possible cases doesn't mean that their judgement is correct and incorrigible. There are several sources of error that tend to undermine the reliability of intuitions about concepts: agents might go wrong in their use of a concept, or be unable to correctly extend it to new cases. Moreover, focusing merely on the classifications that people make neglects the reasons for their classifications of $\mathcal{K}$ s. Ideally, we want to be able to reliably ascertain when a speaker has a competence with a certain concept, and if that competence both explains and justifies their classificatory behaviour. Jackson, and other Canberra planners, argue that we can often empirically determine concept mastery by examining people's production of, and assent to, platitudes about the concepts in question. ${ }^{8}$ Roughly, a platitude is a sentence about the concept which is seen as obvious and undeniable by any competent user of the concept (or by anyone who understands the sentence). It is not required that platitudes be analytic (though not just any believed sentence involving the term will count as platitudinous). The existence of at least some platitudinous sentences follows fairly immediately from the fact that our words have distinctive meanings, which implicitly guide our dispositions to use that word, if we are competent with it. The platitudes express explicitly these implicit dispositions. Who counts as competent will vary from term to term; for many technical terms it will be the commonly 
accepted obvious truths within the relevant community of experts that will count as platitudes. ${ }^{9}$

Using platitudes, we can determine what constitutes a successful analysis on the Canberra plan:

An analysis is successful just in case it gives us knowledge of all and only the platitudes which are such that, by coming to treat those platitudes as platitudinous, we come to have mastery of that concept. (Smith, 1994, 31) Furthermore, we are supposed to be able to turn our platitudes into conceptual analyses (Smith, 1994, 44-7):

1. Start with the platitudes we already have concerning $\mathcal{K}$ :

$$
P_{1}(\cdots \mathcal{K} \cdots), \ldots, P_{n}(\cdots \mathcal{K} \cdots) \text {. }
$$

2. Replace each instance of $\mathcal{K}$ in $P_{i}$ with $X$;

3. Define a $\mathcal{K}$ as an $X$ (or any $X$ ) such that

$$
P_{1}(\cdots X \cdots) \wedge \ldots \wedge P_{n}(\cdots X \cdots) .
$$

The resulting Ramsey sentence describes what it is to be a $\mathcal{K}$ (Lewis, 1970), and we may use it to try to locate $\mathcal{K} \mathrm{s}$ in our fundamental theory. In many cases there will be some concept of the more fundamental theory that satisfies the Ramsey sentence that describes $\mathcal{K}$. The reduction will then have two parts: one conceptual, and one substantive, leading to the reductive conclusion:

Conceptual claim Being a $\mathcal{K}$ involves being an $X$ such that

$$
P_{1}(\cdots X \cdots) \wedge \ldots \wedge P_{n}(\cdots X \cdots) .
$$

Substantive claim $\mathcal{F}$ is the fundamental property which satisfies

$$
P_{1}(\cdots X \cdots) \wedge \ldots \wedge P_{n}(\cdots X \cdots),
$$

(and so makes true $\exists X\left(P_{1}(\cdots X \cdots) \wedge \ldots \wedge P_{n}(\cdots X \cdots)\right)$ ).

Reductive Conclusion Therefore, being $\mathcal{K}$ is being $\mathcal{F}$.

This conclusion 'locates' the property of being a $\mathcal{K}$ within an accepted fundamental ontology: the analysis gives you a set of conditions such that satisfying them implies being $\mathcal{K}$, and that $\mathcal{F}$ satisfies. As far as the platitudes are concerned, $\mathcal{K}$ is coextensive with $\mathcal{F}$. This does not entail that the meaning of $\mathcal{K}$ is $\mathcal{F}$, so while platitudes analysis is certainly compatible with descriptivism about theoretical terms, it does not entail it. For instance, one might believe that 'being $\mathcal{K}$ ' is directly referential if $\mathcal{K}$ is a natural kind term, even while thinking that the things which are $\mathcal{K}$ are nevertheless just 
the things which are $\mathcal{F}$. All the platitudes analysis requires in a direct reference framework is that there is more to competent use than just knowing the referent.

Things are not always so straightforward, for sometimes there may exist no genuine fundamental concept $\mathcal{F}$ that exactly satisfies the $\mathcal{K}$-role, and sometimes there may be more than one such $\mathcal{F}$. An example of the latter might be the example of jadeite and nephrite jade: as far as platitudes involving 'being jade' are concerned, it must be taken to be realised disjunctively at the most fundamental level. The failure of uniqueness still allows location, and so is not as problematic as the failure of existence, when no $\mathcal{F}$ satisfies all the platitudes about $\mathcal{K}$ (as perhaps occurs with the reduction of thermodynamic properties to statistical mechanical properties). Jackson adopts a best-fillers approach: in such a situation, $\mathcal{K}$ is best analysed by that $\mathcal{F}$ (or $\mathcal{F}$ s) that best satisfies the platitudes (or, 'fills the $\mathcal{K}$ role'), if any does.

The two part scheme also allows for conceptual mistakes, despite the relative incorrigibility of the platitudes for competent speakers. This is because those platitudes may not exactly pick out some perfectly natural fundamental property. So despite the fact that our use of the concept does latch on to some perfectly natural property - the best filler of the role - we may still make mistakes, because our criteria imperfectly describe the fundamental property, and will thus misclassify some cases. Further, it could be that no fundamental concept successfully fills the role: there may be no good theoretically respectable candidate explication of the folk concept, and the concept might be jettisoned as belonging to an outmoded conceptual scheme (as perhaps happened with the folk concept of proper function in Aristotle's teleological sense). The Canberra Plan is not a mechanical algorithm, for the second step involves some quite significant theoretical commitments about what criteria identify a best filler, and how tolerant of error a given analysis needs to be. Even philosophers who agree on the platitudes to put in to the Canberra Plan machinery may disagree about which candidates most effectively fill the role picked out by the Ramsey sentence.

An example may help clarify the preceding discussion. Consider the case of colour. There are many obvious platitudes about colour: 'most solid objects are coloured'; 'the colour of an object helps explain how it looks'; 'objects can look different colours under different lighting conditions'; 'the inside of an object doesn't normally affect its colour', and so on. These platitudes are by no means purely analytic truths; on the other hand, they aren't discoveries about colour in any significant sense. Anyone who failed to assent to them would, prima facie, lack some degree of competence with what being coloured involves. We could judge that competence by eliciting judgements about possible cases. For example, if someone claimed that there is a possible situation in which two objects could be the same colour and yet look different to the same observer under the same lighting conditions, we should suggest 
that what they mean by 'colour' (if anything) is not colour, as they have shown a deficit with respect to that concept. Conjoining these platitudes gives us an analysis: colour is that property of objects $X$ such that $X$ helps explain how an object appears and most solid objects have $X$ and .... Given a preexisting ontology, we can then claim that since various surface reflectance properties satisfy $X$, being coloured is just having a surface reflectance property that causes and explains various appearances, etc. The end result, if one chooses this analysis, is a view similar to the so-called 'primary quality view of colour' (Jackson, 1998, ch. 4). Of course things may not be so easy. For instance, maybe there is no fundamental concept that is instantiated exactly when something is coloured, as seems to be the case - there seems no property that explains both how the sky is coloured blue and how someone's eyes may be coloured blue. Maybe colour in translucent objects is fundamentally quite different from colour in opaque objects. In that case the folk concept will be realised disjunctively if at all, and each disjunct may satisfy only some of the platitudes, though in each case sufficiently many to count as an analysis of colour. In any case, this example should give the flavour of a Canberra Plan analysis.

\subsection{A PRIORITY}

Conceptual analysis is traditionally supposed to be a priori, and the Canberra plan version is no different in this respect. However, Jackson (Jackson, 1998, 47-52) and others draw upon some quite untraditional machinery, specifically two-dimensional semantics, to articulate this claim (Chalmers, 1996, 52-71).

Two-dimensionalists argue that the lesson of Kripke (1980) is that some designators have two readings: a rigid reading, on which the designator refers in each possible situation to its actual referent, and a shifty reading, on which the designator refers in each possible situation to what would have been the actual referent, had that situation been actual. This distinction gives rise to two readings of any sentence in which such a term appears; namely, the horizontal proposition which uses the rigid reading, and the diagonal proposition, which uses the shifty reading. The key insight connecting this semantic proposal with a priori truth is Stalnaker's:

An a priori truth is a statement that, while perhaps not expressing a necessary proposition, expresses a truth in every context. This will be the case if and only if the diagonal proposition is necessary... (Stalnaker, 1978, 83)

This may be clarified by an example. The natural kind term 'water' has two readings: the rigid reading, where 'water' refers to $\mathrm{H}_{2} \mathrm{O}$, and the shifty reading, where our word 'water' refers to what the word 'water' refers to in the possible situation under consideration. Another way of describing the shifty reading is that 'water' refers to whatever plays the water role in the 
situation being considered (whereas the rigid reading of 'water' always refers to whatever plays the actual water role, i.e. $\mathrm{H}_{2} \mathrm{O}$ ). On the shifty reading of 'water', the sentence 'water is the thing that plays the water role' is true in every possible situation, and so is necessary. But the proposition expressed (in a given world) by this reading is a priori knowable, because any competent user of the word 'water' knows that their word 'water' refers to whatever happens, around them, to play the water role - no matter what their situation happens to be like. ${ }^{10}$

To apply this to conceptual analysis, we must also give predicates shifty and rigid readings: $P$ rigidly indicates the actual property which is the meaning of $P ; P$ shiftily indicates whatever property would be the meaning of $P$, if the given situation were actual. Once we admit this, it is clear that the conceptual claim, 'Being a $\mathcal{K}$ involves being an $X$ such that $P_{1}(\cdots X \cdots) \wedge$ $\ldots \wedge P_{n}(\cdots X \cdots)$, expresses a proposition that is a priori knowable. This is because if one were a competent user of the concept, no matter what $\mathcal{K}$ s actually were, one couldn't help but understand by $\mathcal{K}$ that property which satisfies all the $\mathcal{K}$ platitudes, regardless of which world at which $\mathcal{K}$ is evaluated. In virtue of knowing how to classify $\mathcal{K} \mathrm{s}$ in any context, one knows that the $\mathcal{K} \mathrm{s}$ are as the platitudes collectively describe; hence on the shifty reading of $\mathcal{K}$, the conceptual claim is necessary and thus a priori.

Though a priori, the conceptual claim about the $\mathcal{K}$ role is impurely a priori, because some experience is required to form the concepts needed to express the claim. However, because any course of experience of sufficient richness would be adequate for this task, no particular a posteriori knowledge is required (Kitcher, 1980). The substantive claim is not a priori, because it requires determinate and particular experiences to determine whether there are any fundamental properties that play the $\mathcal{K}$ role.

The two-dimensional framework is a controversial interpretation of Kripke's conclusions about names and natural kind terms. Soames (2004) argues forcefully that it is nothing other than an ill-considered return to descriptivism. But the claim that the platitudes are (impurely) a priori may, I think, be defended independently of the fortunes of the two-dimensional framework, on the grounds that possession of the relevant concepts puts one in a position to have the right kinds of behavioural dispositions to classify and to assent to and produce appropriate platitudes, and those dispositions are the primary support for the conceptual claim.

\subsection{Modesty}

Conceptual analysis is also traditionally supposed to be modest, yielding no empirical knowledge, and Jackson (1998) concurs (43). The a priori status of analyses seems to support modesty, because a priori truths are not empirically contentful. (Even contingent a priori truths [like Kaplan's 'I am here 
now'] aren't substantively empirical, because they aren't tied to any particular experience of a substantive state of affairs.)

But there is an apparent tension here, because some platitudes are existentially quantified, and the analyses that result from Ramsifying these platitudes cannot be modest. Thus it seems that, very plausibly, there are ontological commitments that are taken on when concepts are learned, so that in some cases we wouldn't count as adequate users of the concept if we failed to assent to some arguably a posteriori existence claims.

Several apparently platitudinous existential claims present themselves. Lewis claims that it is a platitude about modality (it is 'uncontroversially true') that things needn't be the way they happen to be, and that we can acceptably paraphrase this claim as 'there are many ways things could have been besides the way they actually are' (Lewis, 1973, 84). In the case of colours, there are platitudes like 'red is more similar to orange than blue' (Smith, 1994, 29), which seem straightforwardly to entail that something is more similar to orange than blue. This claim would not be platitudinous if we had no colour-related experiences sufficient to give us mastery of the concept, or if there were no colours (and colour eliminativists - those who think the best account of the colour platitudes is that nothing fundamental answers to them -must and do deny it on that basis). Its existential commitment is understandable, but no less committing because of that. These immodest platitudes undermine the reliability of conceptual analysis, for very few people are willing to accept the existence of possibilia even if our ordinary ways of talking seem to presuppose their existence.

But one needn't accept that these platitudes are genuinely immodest. In fact, proper attention to their role and origin suggests a kind of (hermeneutic) fictionalism about the existential commitments of platitudes (Yablo, 2001; Rosen, 1990). Platitudes simply express the rules that govern the correct use of a particular bit of discourse, and should, if possible, be given a literal reading. Yet, although the discourse works as if the immodest platitudes were true, the naturalistic explanation of the origin of the discourse cannot lie with the objects presupposed by those platitudes. Whatever the natural origins of modal language, it couldn't have been in response to our ancestor's sensory awareness of a realm of mere possibilia. Regardless, modal discourse is systematic and regular, and the commonly accepted principles are accurately captured by Kripke semantics. If modal reality is radically different from how our modal language depicts it, it is not clear that we have any access to facts about that reality which could enable us to 'correct' our discourse. As it turns out, modal language would still be best systematised by standard Kripke semantics regardless of how modal reality turned out. ${ }^{11}$ Similarly, the origins of our concept of red in perceptual experience as of red objects is obvious, but the mere fact that our colour discourse has an easily available naturalistic explanation does not yet commit us to the existence of a quality 
to which the predicate 'is red' corresponds. We may regard the phenomena of perceptions of red as best systematised by a discourse that introduces 'red' as a quality term applying to external objects, regardless of whether such a quality exists.

The upshot is that for platitudes about colour or modality to function correctly in regulating our use of the concept in discourse involving 'red' or 'possibly', it needn't be that the literal content of those platitudes is true. Correspondingly, endorsement of the platitude needn't be interpreted as an assertion of the literal content. The best way to understand this is that these apparently possibilia-presupposing bits of discourse function instead as regulatory assumptions, introduced in order to facilitate and aid the expression of more prosaic and more important judgements about causes and hypothetical situations (and common perceptual experiences). The adoption of the hypothesis of possible worlds, and the hypothesis of properties, may be entirely explained by the fact that adopting them makes expressing other claims easier, while remaining indifferent concerning the real existence of possibilia or qualities.

This hermeneutic fictionalist reading of the platitudes remains perfectly compatible with the platitudes being literally true, although further philosophical argument is required to establish that an investigation into the correct rules of language does indeed give rise to genuine truths. In the case of modest platitudes that just relate one concept to another, we can argue straightforwardly that the method of classification of possible cases tells us under which conditions that concept applies, and therefore which conditions are involved in an analysis of that concept. For modest platitudes, therefore, there is a strong presumption that the way that language works gives a reliable guide to how the concepts are to be understood - so modest platitudes are both correct rules and literally true. But this argument is not available in the case of immodest platitudes, simply because of the observation that language could function in precisely the same way, governed by the same platitudes, and yet the existential presuppositions be false. Existentially quantified platitudes thus should be read as merely quasi-asserted, which allows that conceptual analysis can be modest even if it includes such platitudes. Note that this division of platitudes has no significance for the analyses we end up giving, for both kinds of platitudes have the same significance and role in the Ramsey sentences governing the concepts. I shall return to these issues, in the case of mathematical existence, below ( $\$ 6)$.

\section{Mathematical Axioms and Conceptual Analysis}

We're now in a position to put our Canberra Plan account of conceptual analysis to work, and sketch an account of mathematics as conceptual analysis. 
The broad outlines of the view are as follows. We have an intuitive grasp of the concepts of (some fundamental parts of) mathematics. Those intuitions are articulated in platitudinous remarks involving those concepts, particularly concepts like 'set' and 'number' which have significant use and role outside of mathematics; the platitudinousness of the remarks serves to verify the truth of the intuitions. Ramsification over those platitudes gives us a long and complicated description of the concepts. Accepting Gödel's remark that the axioms of mathematics merely unfold the concepts, it is natural to understand the axioms as the simplest, best, most compact, and most systematic description of the concepts. Given our mastery of the relevant mathematical concepts, we can know the axioms to be a priori true. A substantive analysis will then go further, and identify natural properties that best play the role delineated by the axioms, if any.

\subsection{Analysing Mathematical Concepts}

What might our mathematical platitudes be? The best place to look, it seems, are the introductory remarks made about the concepts, preliminary to any substantial mathematics being done. At least in the cases we're interested in, these preliminary remarks serve to do more than give an informal introduction to the concepts (as might be there sole purpose in the case of more obscure mathematical concepts, like a group), but also serve to prepare one, in terms of concepts one already possesses, to understand the new concept.

Some platitudes about numbers might include platitudes about the application of numbers: 'numbers can be used to count objects'; 'there is at most one number associated with any group of things'; 'the number associated with a group of things doesn't depend on the kind of things they are'; 'positive whole numbers ... give the answer to the question "How many?"' (Frege, 1884, 5). There are platitudes about the relations of numbers to each other: "numbers are ordered'; '7 is bigger than 5'; '7 is closer to 5 than it is to 3 '. There are platitudes about the objectivity of mathematics: 'if two people disagree about a fact about numbers, at most one of them can be right'; 'arithmetical rules can decide mathematical disputes'; 'arithmetic would still be the same even if the things in the world were quite different'. There are platitudes about how we learn numbers: "if you want to teach someone a number word, like "seven", then show them many collections of 7 objects'. There are also platitudes about the arithmetical operations: 'addition is closely associated with combining two groups of things'. These sentences by no means exhaust the platitudes about natural numbers and arithmetical operations on natural numbers, but they should be enough to establish that there is a rich set of platitudes surrounding our use of numerical and arithmetical concepts in perfectly ordinary contexts. 
Each of these claims, I suggest, should strike us as completely uncontroversial, or platitudinous, at least with respect to correctly characterising the concepts involved. Antirealists may reject the platitudes, but only by acknowledging that they correctly describe a concept that is not, as it happens, instantiated (Field, 1980).

Having gathered the platitudes, we can apply exactly the method of Ramsification that the Canberra Planners advocate for conceptual analysis, obtaining a sentence that characterises the mathematical concept in question: 'being an $M$ is being an $X$ such that $P_{1}(\cdots X \cdots) \wedge \ldots \wedge P_{n}(\cdots X \cdots)$ '. As before, these platitudes are supposed merely to unfold the implicit concept associated with that mathematical term; as such, the resulting conceptual claim should be a priori true, knowable as such by any competent user of the mathematical concept.

However, this list of platitudes will likely be extremely long, and extremely repetitive. We can reduce the difficulty of handling such unwieldy lists of platitudes, by axiomatising the concepts.

Definition 1 (Axioms for a Concept). $A_{1}, \ldots, A_{n}$ are axioms (or axiom schemata) for a concept $\mathcal{M}$ iff $\left\{A_{1}, \ldots, A_{n}\right\}$ are a simple and concise set of independent claims (or claim schemata) that entail the core platitudes about $\mathcal{M}$, and do not entail the negation of any core platitude about $\mathcal{M}$.

This explains Gödel's remark that 'the axioms force themselves upon us as true': not because we have some mysterious faculty, but for the wholly unmysterious reason that they are completely platitudinous in content. However, the axioms needn't be platitudinous in form: no axiom need appear in $\left\{P_{1}(\cdots X \cdots), \ldots, P_{n}(\cdots X \cdots)\right\}$. This explains why axiomatisation is not trivial, because it can be quite difficult to decide which set of claims is genuinely simple, and genuinely captures all of the platitudes about the concept in question. Of course, in general we won't check any putative axiomatisation against some pre-existing list of platitudes; rather, we'll take an implicit and intuitive understanding of the concept, and produce axioms directly for the concept. The ultimate check on the axiomatisation, though, will be whether it does really entail claims that are platitudinous with respect to the concept in question. Again, it is our dispositions to assent to the platitudes that count; our axioms are directly sensitive to these dispositions, and are later tested by whether they yield platitudinous claims.

Not every case is this simple, however. As in any case of conceptual analysis, there may be failures of existence and uniqueness of the concept described by the platitudes. Frege famously put forward a number of platitudes about the concept set, including the platitude that 'for any predicate, there is a set of things satisfying the predicate'. The naive ('platitudinous') set theory that he formed on the basis of these platitudes was inconsistent, so that no coherent concept of set was described by all and only those plat- 
itudes. The solution to this problem is inegalitarianism about platitudes. On this view, some platitudes are privileged, either through being more central to the concept (the real kind) we are trying to explicate, or through being platitudinous about a closely related, consistent concept. ${ }^{12}$ We then generate axioms that capture the closest consistent concept in the vicinity of the original set of platitudes; the one that best makes the central platitudes both true and platitudinous, while avoiding the inconsistency. So ZFC set theory gives a consistent concept of set that best captures the inconsistent intuitions that went into naive set theory. (Type theory gives another consistent set concept, but one that mathematical consensus seems to suggest is a less intuitively plausible reconstruction.)

Gödel himself argued that the best account of ZFC set theory rests on a different set of platitudes - the so-called iterative conception of set (see Boolos (1971) for a clear articulation of the relevant platitudes):

a set is something obtainable from the integers (or some other welldefined objects) by iterated application of the operation "set of", ${ }^{\alpha}$ not something obtained by dividing the totality of all existing things into two categories...

${ }^{\alpha}$ This operation ... cannot be defined satisfactorily, but can only be paraphrased by other expressions involving again the set concept, such as: "multitude of $x$ 's", "combination of any number of $x$ 's"...

(Gödel, 1964, 474-5)

So it is pre-mathematical platitudes about multitudes and collections that ground the concept of set, platitudes that are paraphrases of claims involving the concept set, and which are themselves captured by axioms of ZF set theory.

We can also have failures of uniqueness. Arguably this is the situation with respect to the concept of parallel line in geometry. Euclid's fifth postulate is independent of the postulates; though the other postulates capture the concepts of point and line, they don't do so in such a way as to uniquely answer the question of when two lines are parallel, which needs to be added in as an additional constraint. Famously, this can be done in more than one way, giving rise to various alternative ('non-Euclidean') geometries. The situation is that the concepts of point, line and parallel aren't individually rich enough to determine their relation to each other. Thus the concepts we have can be extended, fixing in different ways the relations between them. This case is quite a nice example, because it was long recognised that the fifth postulate was quite different to the other four; from our perspective, the difference is that the other postulates are platitudinous ("between any two points there is a straight line'), while the fifth postulate is not platitudinous with respect to 'point' and 'line' (though it may be an intuitive platitude about parallel lines, pre-theoretically). In this case, we have a problem, because it is clear that though the other four postulates do pick out a family of concepts characterised by the theory of absolute geometry, there are too few constraints 
on these concepts for them to be of any use. It is only through extrinsic links with other concepts, and through the concepts coming to be used in various physical and folk theories, that we have enough data to decide which of these concepts should be applied; it remains the case that all the concepts are of equal validity, from a conceptual or mathematical perspective. The first four postulates are true of each concept that satisfies the platitudes, while the various fifth postulates characterise the various mathematical natural kinds. ${ }^{13}$ This liberal attitude is vindicated by contemporary mathematical practice.

This example is illustrative in another respect also. One thing that drives the supplementation of absolute geometry with one of the various competing parallel postulates is a desire for completeness. That is, a given genuinely natural concept should wholly characterise, insofar as that is possible, the truths of a domain of discourse. The 'meta-platitude' that the axiomatisation should wholly characterise the subject of the platitudes then drives conceptual analysis even in the case of non-Euclidean geometries and other prima facie counterintuitive mathematical theories. The particular role of conceptual analysis here is that the resulting articulated and axiomatised concepts should satisfy, by and large, the pre-theoretic platitudes; since one of those platitudes is that the concepts should be natural and complete, several articulated concepts might equally count as acceptable even though not all are platitudinous in every respect, and one is 'more acceptable' than the others. It is fairly clear that ordinary Euclidean geometry is more widely regarded as platitudinous, and non-Euclidean geometry is regarded as counter-intuitive in many respects. That may well be true, but that the first four axioms still force themselves upon us as true in every competing geometry shows the platitudinousness of those axioms. And the desire for completeness then forces another axiom upon us, even though various axioms turn out to be possible completions. Which axioms are most platitudinous will have something to do with the structure of empirical experience, which looks Euclidean in many respects. What this example shows, I think, is that the vagaries of empirical experience may well be smoothed out by the flexibility of most genuine mathematical concepts to stretching and extension.

A similar situation is perhaps seen with respect to the concept of a polyhedron in Lakatos' famous discussion of Euler's theorem that $V+F=$ $E+2$ (Lakatos, 1976, 88-92). In this case, the intuitively plausible claim is that Euler's theorem holds, because it does for the most obvious class of polyhedron. But as the concept was further investigated, many independent characterisations of the concept were seen to yield different, though similar, results. Interestingly, these other concepts of polyhedron were not taken to be counterexamples to the original theorem, but rather to illuminate different kinds of polyhedron, and to provide new grounds upon which to theorise. This process, which Lakatos called 'concept-stretching in response to proof and counterexample', looks a lot like sorting through and refining various 
competing platitudes to discern which concepts they are legitimately associated with. The decision as to which kind of polyhedron is a natural kind is one that is best made in light of the uses to which the concept is to be put.

Indeed, Lakatos (1976) can be read as an extended exploration of the primacy of conceptual questions over ontological questions in driving mathematical advances. As such, there are a great many apparent affinities between his account and my own. The Lakatosian ideas that proofs are akin to thought experiments, and that mathematics might be driven by quasi-empirical reflections on concepts, are both quite germane to the present account. But I am wary of aligning myself too closely with Lakatos. His view of what a concept is by no means the same as the platitude-driven concepts I appeal to; and the Canberra Plan machinery would be quite foreign to Lakatos' framework. The similarities between my account and his are suggestive; I'm not prepared to say how close the accounts really are.

\subsection{Virtues of the Present Account}

The present account has several virtues from a philosophical perspective.

\subsubsection{Phenomenology}

Firstly, it makes sense of the phenomenology of foundational mathematics with what it feels like to do mathematics. Since what we do as philosophers of mathematics is, in large part, to interpret the practices of mathematicians, this connection surely makes the present account more plausible (just as it makes proposals like formalism less plausible, simply because they seem to make little sense of what mathematicians regard themselves as doing). The primary phenomenological fact is that mathematics places a great emphasis on exploring the properties of certain kinds of mathematicalia, an exploration that derives not from empirical engagement with these objects, but by examining the concepts that define the kinds of mathematicalia that there are. As a part of mathematics, this emphasis on concepts isn't in any way decisive for mathematical ontology, but contemporary mathematics is remarkable for its emphasis on mathematical concepts rather than mathematical objects an emphasis the present account can readily explain.

\subsubsection{A Priority and Necessity}

The present account also explains another puzzling aspect of mathematics: the apparently a priori nature of mathematical claims. The axioms have an a priori status because collectively they express simply claims about concepts - claims like 'being a square is to be the kind of thing to which we'd apply the concept square'. As I discussed earlier ( $\$ 3)$, that kind of sentence is true whenever it's uttered, because it follows simply from an understanding of the concept of square (even though it needn't be necessary, and we don't 
have to regard square as response-dependent). Jackson argues that in the case of square, the two-dimensional profile collapses, and in such cases we have a priori knowledge of metaphysical necessities (Jackson, 1998, 49). If this is true of mathematical concepts more generally, then we also have an explanation of the necessity of mathematical claims, as deriving from the fact that these concepts have a modally invariant intension.

\subsubsection{Epistemology}

Our interpretation of mathematical practice need not preserve mathematicians' own interpretations of their practice, which have tended to fare rather less well than the practice itself. In particular, our interpretation can be sensitive to particularly philosophical scruples about epistemic access - scruples that, if they concern mathematicians at all, seem to lead them into hypotheticalism or formalism rather than more sensible interpretational stances. Here the present account does rather better, because we have an empirically acceptable story to tell about the origins of mathematical platitudes. In the case of number platitudes, it seems clear enough that we learn number concepts through empirical experience with operations on finite collections, and our number platitudes systematise and regularise facts concerning these experiences. ${ }^{14}$ Kitcher (1978) articulates a similar thought when he says "mathematics in general is a system, like geometry, which we apply to explain our experience" (134). In particular, Kitcher and I agree that it is our experience with collecting and correlating finite sets that is the ultimate source of our mathematical concepts. These concepts are idealised, certainly, and how we get from the messy details of empirical experience to the cleaned up and idealised concepts is a tricky question. Nevertheless we clearly do manage to do so; and having done so it is fairly easy for many of us to recognise platitudinous articulations of those mathematical concepts. For example, platitudes about relative magnitude of numbers and sets can easily be generated once a language learner is in possession of the concepts of collections and correlations. In light of all this, I must slightly modify our earlier observation, and maintain that mathematics is impurely a priori. That is, some course of experience sufficient to inculcate the mathematical concepts is necessary — but any such course of experience will do.

\subsubsection{Applicability}

The present account also has some potential to help with the difficult and puzzling issue of the applicability of mathematics. As the above remarks make clear, the axioms of arithmetic and set theory emerge from our everyday platitudes about collections and operations on collections. As such, it is not at all surprising that those branches of mathematics should turn out to be applicable to these areas of experience. It is certainly far less surprising on the present view than on a platonist or nominalist understanding of mathem- 
atical truths. In these areas, at least, the grounding of conceptual intuitions in experience has the potential to explain the applicability of the body of truths extracted by the Canberra plan machinery from those concepts. The sense in which mathematical truths are a priori is similarly not incompatible with the quasi-empirical nature of mathematical truth that follows from the present view.

Yet the problem of applicability is not wholly solved. In common with other views that stress the empirical origins of mathematics, the present view suffers from two difficulties. The first, that the content mathematics seems to outstrip the content of experience, will be dealt with in the immediately following section (\$4.2.5). The second is that, while the applicability of mathematics to familiar areas of experience (from which it was initially generated) is explicable, there is the puzzling phenomenon of novel applications, which show that the mathematical concepts can be applied in a new and unexpected way. The applicability of the concepts in this framework cannot be expected to follow in any straightforward fashion from the platitudes about how that concept fares in other applications. To this problem I have no full solution, but perhaps one might appeal to the further empirical fact that nature is uniform in some sense. That is, truths about one part of nature, if sufficiently general, will apply to other parts of nature. The mathematical truths extracted from empirical experience of collections are non-specific and topic neutral: they apply to collections of any kind of object, for example. So we might hope that this observed non-specificity, when combined with the uniformity of nature, might give rise to genuine generality in our mathematical concepts, and these very general concepts might well apply in novel situations. That, anyway, is the hope; it is at least a direction of inquiry that is open to the present account. Even if it is not convincing, that shouldn't count too strongly against my proposal, for no rival Platonist or nominalist account has much more to say about this difficult problem.

\subsubsection{Fruitfulness}

We also have a way of understanding extrinsic justifications in mathematics. It is true that mathematicians do often talk as if the axioms were self-evident, and in large part they must be as they are fairly directly generated by platitudes about the concepts in question. But one also sees axioms justified on the basis of their fruitfulness, or non-restrictiveness - extrinsic justifications based on the consequences adding that axiom would have for the body of accepted mathematical claims:

A probable decision about [an axiom's] truth is possible ... by studying

its ... fruitfulness in consequences. (Gödel, 1964, 477)

Here, I take it, we can read 'fruitfulness' in one of two ways. On the one hand, we can read it as fruitful in enabling us to prove many desirable mathematical theorems. On the other, we can read it as fruitful in producing an interesting 
and rich universe of mathematical objects. There is a tension between these two readings, because a rich and interesting conception of set, for example, that gives rise to many interesting kinds of arbitrary set, often prevents global and wide ranging theorems to be proved about all sets. Axioms that are fruitful in the sense of proving lots of strong theorems tend to do so only by restricting or minimising the diversity of the set theoretic universe. ${ }^{15}$ Though Gödel showed some early fondness for the axiom of constructibility $V=L$, he later comes to reject that axiom as inconsistent with the unrestricted and open spirit of the iterative conception of a set (Gödel, 1964, fn. 19, 478-9). In this judgement later set theorists unanimously concur; $V=L$ was taken to unduly and arbitrarily restrict the universe of sets, and was to be rejected for that reason (Maddy, 1997, ch. 6).

We can begin to understand this if we remind ourselves of how the platitudes give rise to the axioms. Just as in the non-mathematical case, our platitudes aren't incorrigible. There is a constraint placed on any platitudes analysis that it be systematic: that it make all the platitudes mentioning numbers fit together in such a way that it is plausible that those platitudes are about numbers, so that we can be confident that those platitudes describe the genuine natural concept of number. Our axioms in some sense explain our platitudes, by unifying them under one systematic conception (this is another example of inegalitarianism about platitudes). A particularly neat axiomatisation might in itself be taken as evidence that there is an elegant and attractive mathematical concept being articulated, and, even though such considerations are somewhat syntactic, might be taken as evidence that some of the platitudes may be sacrificed for elegance.

Having made this observation, we are in a position to understand why fruitfulness and unrestrictiveness might be relevant considerations in favour of an axiom. A fruitful axiom, in the present sense, is one that doesn't arbitrarily restrict the kinds of mathematical objects that there are. This doesn't provide certain evidence of the truth of the axiom, but it makes it more likely that one hasn't made in one's axiom an arbitrary assumption that unduly limits the extension of the original concept. The thought is that the platitudes say nothing to decide such cases, and as long as the new axiom is in overall harmony with the original platitudes, an unrestricted conception is more likely to do justice to those platitudes. This is especially so in the case of iterative set theory, where the original intuitive vision itself contains some commitment to arbitrary expansion, so that unrestricted axioms are arguably more in the spirit of those original intuitions. At this point our observations about systematicity have a part to play, because, with respect to the iterative conception of a set at least, unrestrictedness is an indicator of the naturalness and systematicity of the concept. In other branches of mathematics, we have a similar pattern. Consider the extensions of the concept of number beyond natural numbers to the reals, and then to complex numbers (or consider the 
extension of the concept of function to the present notion of an arbitrary function). In response to novel examples, the concept is extended and stretched; its responsiveness to, and pliability under, such stretching is a relatively reliable indicator of the naturalness of the concept in question. Extrinsic justifications of axioms do not suppose that fecundity is truth-conducive in itself. Rather, fruitfulness is reliably correlated with the most natural understanding of the concepts, without arbitrary restrictions that do not derive from any platitude. As Yablo puts it, mathematics seems to obey the following methodology:

(A) to articulate the clearest intuitive conception possible, and then,

(в) subject to that constraint, let all heck break loose. (Yablo, 2005, 109)

\subsubsection{Connections to Philosophy}

Finally (though this may be considered an objection by some), the present proposal explains the considerable methodological similarities between philosophical and mathematical practice. Both mathematics and philosophy are relatively insensitive to empirical considerations, except insofar as the empirical sciences give rise to problems of a mathematical or conceptual nature. The apparent dissimilarities — that mathematics is certain, while philosophy is anything but - are explained by pointing out that mathematics is a special case of philosophical inquiry. In particular, mathematical discourse is much more regulated and narrow than the more unruly discourse that philosophical inquiry is generally concerned with. There is a consequent unanimity in judgements about possible cases, and this in turn makes the axiomatic method possible in mathematics. Yet, as disputes about the correct way to analyse mathematical concepts like entailment or polyhedron show, the difference is not of kind, but of degree.

\section{Objection: Consequences of Generality}

The generality of mathematical platitudes played an important role in several of our observations above, for example in explaining why any course of experience would be sufficient to provide one with the mathematical concepts, and in, tentatively, explaining novel applications. Yet this generality appears to have two severe costs in the Canberra Plan framework.

Firstly, the generality of the concepts in question might mean that when we come to Ramsify over the collection of platitudes, we end up Ramsifying practically everything, and thus end up characterising nothing. In response, the adherent of the Canberra plan in the area of mathematics will have to abandon the quest for reduction. As we saw, Jackson uses conceptual analysis to locate the terms of one theory in another theory. If we regard the second theory as more fundamental than the first, then we should regard this location as a reduction. Yet if we follow this conclusion to its natural end, it seems 
we will successively move to theories with more and more impoverished vocabularies. Yet for some concepts, and the concept of set is a good example, there seems to be no more basic vocabulary. Recall Gödel's remark (quoted above on p. 15) that the 'set of' operation cannot be analysed, only paraphrased by other expressions that again involve the concept of set. This seems to indicate that a wholesale reduction of every mathematical concept, all at once, to non-mathematical concepts, may not be possible. So we must give up on one part of the Canberra plan project with respect to mathematics. Nevertheless, we can keep the parts of the story that involve platitudes and our empirical access to them. We can reduce other mathematical concepts to the privileged concepts of set and number that have a significant use outside of mathematics (and it is that use that generates non-trivial platitudes about these concepts). And we can effect a piecemeal reduction for mathematical concepts in succession, locating each such concept with respect to other concepts (including some mathematical concepts), even if we cannot effect a wholesale reduction. This was to be expected: the reductive aspirations of the Canberra plan were to locate autonomous pieces of discourse with respect to the rest of the language. The concept of set seems so widespread that there is no easily corralled part of the language that is the natural home of that concept. So reduction looks implausible, even while the articulation of the concept via platitudes and the resulting axiomatisation can still go through. And, of course, the substantial reduction of the platitude-articulated role to its realiser remains possible (though see $\$ 6$ ).

Secondly, the platitudes about numbers, for example, will apply to any collection of objects that can be arranged in an $\omega$-sequence, and thus the platitudes will fail massively to secure uniqueness, in the sense that many collections of objects will equally satisfy the conceptual role articulated by the platitudes. This certainly has consequences for the substantive reduction of mathematical properties to fundamental properties, as the extension of the mathematical relations will be massively underdetermined by the platitudes. This is good in one way; all we need in many cases is to realise that something plays the number role, and is characterised by the platitude-grounded axioms, from which follow various mathematical truths. But no substantive identification of mathematical properties with more fundamental properties is available, because any such identification will go beyond the content of the platitudes. (This should perhaps remind one of the Julius Caesar problem for Frege.) Since we've already abandoned the wholesale reduction of mathematical discourse to non-mathematical discourse, this is perhaps not so worrying. But nevertheless the idea that there are mathematical structures characterised by the axioms and apparently about objects, yet unable to secure reference to those objects, is somewhat puzzling. We can perhaps try the piecemeal reduction strategy, and locate mathematical objects uniquely by fixing the referents of other mathematical terms, yet this strategy is unsatisfying. One 
possible move here is a kind of structuralism, which would claim that the identities of the mathematical objects is not important as long as they satisfy the platitudes. I'm tempted by another alternative, a kind of fictionalism about mathematical objects. This move similarly undermines the significance of the failure of uniqueness, and solves another difficult issue which we must now confront.

\section{Objection: Existential Platitudes}

There must be some disquiet at this point about the seeming immodesty of some of the mathematical axioms. The basic issue is simply that some axioms are existentially quantified, like the axiom of Infinity, which states just that there exists an infinite set. But, as I suggested earlier (\$3.2), it seems wildly improbable that we could derive such strong conclusions by conceptual analysis alone. At best, the objection continues, conceptual analysis could give you a hypothetical characterisation of the mathematical realm: were such concepts to be instantiated, then the universe of (sets, numbers,...) would look as the axioms say.

On the basis of this, it is tempting to separate the axioms into two groups: the purely conceptual claims, which are true, and the ontologically committing claims, which are not yet known to be true or false - and only accepting the former. Yielding to this temptation would, I suggest, be a mistake. The axiom of infinity is not some kind of optional add on to the concept of a set. If the hierarchy were cut off before collecting all the finite sets, we would have a restrictive set concept that doesn't correspond to the intuition driving the iterative conception, namely that any finite cutoff in the number of permissible iterations of 'set of' is an arbitrary restriction of the concept. Qua platitudes, there is no distinction between the ontologically committing and the ontologically neutral claims; by making such a distinction we distort the concepts we were attempting to analyse.

It is not inconsistent to take these existentially committed claims as truthapt, but it does seem to me to rest upon a misunderstanding of the use and point of mathematical discourse. Consider again how we learned mathematical language as we learned how to systematically discuss - and manipulate - finite collections of objects. Mathematical concepts originate in experiences of representing the content of certain awkward and otherwise unmanageable claims about real-world collections. Of course the higher reaches of set theory and even arithmetic don't serve any such real world purpose: yet they are, as I observed above, constrained by the concepts formed in response to everyday small finite cases.

This all tends to suggest that the correctness conditions for mathematical claims should be understood as indicating the extent to which those claims 
succeed in articulating and developing the conceptual structure developed to serve the everyday purposes of counting, grouping and adding. One couldn't understand the development of those conditions in any other way. As such, the correctness of the ontological claims must also be seen in the same way: those claims are correct just in case they serve to best articulate the conceptual structure suggested by the most basic areas of small finite arithmetic and set theory.

However, it is no part of those practices that the only numbers must be small, and the only sets finite sets of concreta. Indeed, precisely the opposite is true of the concepts we form in response to early mathematical experiences: because we learn these concepts by generalising over a large range of cases, we form concepts that are unrestricted with respect to the size of the numbers or sets. Moreover, as is especially evident in the case of iterative set theory, we form a concept of set that both functions as an aid to the representation of facts about finite collections of concreta, but also as an object of set theory in its own right.

The existential axioms of set theory or arithmetic must thus be understood as claims put forward in a context quite different from the one that consideration of existential claims ordinarily suggests. That is, these are not claims put forward with the purpose of describing fundamental ontology, but rather as best systematising an concept which is indispensable in the rest of our activities. As such, it would be incorrect to interpret those existential claims as if they purported to describe mathematical reality. Rather, we should interpret those claims as asserted with a different purpose entirely: to systematise and regulate our dealings with the entities to which mathematics is applied.

The position that this line of reflection suggests is thus a kind of fictionalism about mathematical objects, just as in the cases of conceptual analysis I examined earlier (\$3.2). ${ }^{16}$ The fictionalist regards apparently ontologically committing mathematical claims as not actually asserted, but rather regards them as uttered merely to articulate an practically indispensable conceptual framework. The best interpretation of mathematical language doesn't take it to aim at making assertions about the nature of mathematical objects; its point all along was quite different. ${ }^{17}$

The apparent form of the axiom of infinity suggests that the sets, considered as a special type of object, are of primary interest to the mathematician, and from this the special epistemic problems of platonism emerge. If we pay due attention to the point of mathematical discourse, it can quickly be seen that the concern with mathematical objects is an artifact of the surface appearance of mathematical assertions. On the conceptualist account I illustrated above, mathematical discourse could go on quite as well, with the same empirical foundation, and the same applicability, regardless of the ontological status of mathematical objects. 
The significance of mathematics comes from the fact that it articulates a conceptual structure that is highly applicable to natural science and everyday interaction with discrete concreta. That our language best expresses that conceptual structure by use of existential quantifiers is no reason to regard the mathematical objects as the primary subject matter of mathematics, rather than the mathematical concepts which the present account suggests are genuinely primary. The apparent necessary existence of mathematical objects, for example, is a fairly direct consequence of the conceptual necessity of the axioms, and that necessity can thus be explained away - it is not a surprising and deep fact about mathematicalia, but a trivial consequence of the fact that we cannot conceive of applicable mathematical concepts that are otherwise than as they actually are.

\section{Conclusion}

I've argued that natural thoughts on the special role of intuition in set theory and arithmetic can be fruitfully combined with the Canberra Plan view of conceptual analysis. This yields an interesting view that foundational mathematics just is conceptual analysis, and strongly motivates a certain kind of fictionalist approach to mathematical objects, at least those of fundamental mathematics. This may provide at least a starting point for mathematical epistemology more generally; at least it seems to me to give a plausible account of the special epistemic status of these branches of mathematics and how they could be known.

Though I find this view quite attractive and defensible, I'm not yet convinced. I stress that I haven't tried to show any more than this conditional thesis: the Canberra Plan view of conceptual analysis and a priori knowledge, taken with some plausible views about the role of intuition in mathematics, together suggest a quite attractive epistemology for foundational mathematics. They also suggest a metaphysics of mathematics that is independently promising and that dovetails nicely with recent concerns. Given the continuing debate over Benacerraf's objections to Platonism, new and promising accounts of mathematics should be welcomed and explored, and I recommend the present proposal on that ground at least.

\section{Acknowledgements}

Thanks to Brian Ball, Lara Buchak, Kenny Easwaran, Øystein Linnebo, Tim Williamson, anonymous reviewers, and an audience at a meeting of the Philosophical Society in Oxford. 
Notes
1 'Mathematical intuition need not be conceived of as a faculty giving immediate know-
ledge of the objects concerned' (Gödel, 1964, 484).
${ }^{2}$ Charles Parsons has long been at pains to emphasise the significance of conceptual intu-
itions in Gödel's thought (Parsons, 1995, 65-6).
${ }^{3}$ This does not capture all the subtleties of Gödel's position. For example, Gödel seems
elsewhere to emphasise that the axioms of mathematics can be given an extrinsic justification
in terms of fruitfulness of consequences, and there is some tension between this latter position
and the idea that axioms unfold the intrinsic content of mathematical concepts - see $\$ 4.2 .5$.
${ }^{4}$ For a more detailed examination of the Canberra Plan, see Nolan (forthcoming). Its main
themes are lightheartedly outlined in the 'Canberra Planner's Credo' (Nolan, 1996). (The
origin of the term 'Canberra Plan' is somewhat more disparaging (O'Leary-Hawthorne and
Price, 1996, fn. 23).)
5 Really, these will be 'interpreted' models, in which the set-theoretic structure is taken to

${ }^{5}$ Really, these will be 'interpreted' models, in which the set-theoretic structure is taken to denote properties and relations.

${ }^{6}$ This is a kind of global supervenience (Stalnaker, 1996): all the properties of $T_{1}$ are able to be mapped exhaustively onto properties in $T_{2}$ in such a way as to make all the claims of $T_{1}$ true, without thereby committing ourselves to the explicit definability of $T_{1}$ in purely $T_{2}$ terms.

${ }^{7}$ Thus undermining the non-reductionism championed by some defenders of global supervenience.

${ }^{8}$ Yet someone who cannot articulate into platitudes the content of a given concept may still perform admirably on the task of classifying possible cases, and thus count as possessing the concept. Though Jackson suggests that all competent users of a concept need to share its core platitudes, the picture is not so crude as to demand that 'sharing' involves carrying around a common creed in the head, though certainly agents who actively dissent from the platitudes should not count as possessing the concept. I don't think that the proposal must involve 'understanding-belief links' (Williamson, 2006); all it needs is minimal 'possessionbehaviour' links.

Nevertheless, the view does conflict with proposals about concept possession that sever the link between competent classificatory and linguistic behaviour with respect to a given word, and having a concept to which that word corresponds. If agents can have behaviourally invisible concepts, or can fully understand a word without possessing the associated concept, then this project will fail. There are certainly debates over such matters (Williamson, 2006), but I cannot engage deeply with them here, and must hope that many will find the Canberran conditions on concept possession and mastery plausible enough.

${ }^{9}$ It is a matter of controversy just what counts as a platitude; for an account of this debate, and a tentative defense of the middle-ground position I defend, see Nolan (forthcoming).

${ }^{10}$ Of course perhaps some statements are not entertainable (perhaps too long or complex). Hence even if they are a priori true in Stalnaker's sense, they aren't knowable. If we restrict our attention to entertainable sentences, those that are a priori true in the present sense are arguably a priori.

${ }^{11}$ Just as the account of counterfactuals that Lewis proposes is widely accepted as descriptively accurate to our practice, despite controversy over how its semantics are to be mapped onto reality (Lewis, 1973).

${ }^{12}$ It is interestingly not clear quite where on the conceptual/substantive divide one should place this problem. If one believes that there can be inconsistent concepts, then one should say that, nevertheless, there can be no natural property that is inconsistent, and the inconsistency is a failure of a concept to pick out any perfectly natural property. On the other hand, if con- 
cepts must be coherent to be legitimate, there is no inconsistent set concept; just inconsistent, apparently platitudinous claims about sets (perhaps they are platitudinous claims about two different set concepts, unfortunately mixed up and confused).

${ }^{13}$ In so-called 'spherical geometry', another axiom (governing the length of lines) must be altered to avoid contradiction.

${ }^{14}$ McGee (1997) gives a sketch of a similar thought in an ultimately different metaphysical framework. Feigenson et al. (2002) give details on how children seem to acquire their basic aptitude with number concepts, in a way that seems empirically possible and not to presuppose any strong thesis of epistemic access to the numerical objects.

${ }^{15}$ In the extreme case, a contradiction is extremely fruitful in logical consequences, though at the cost of emptying the universe of objects.

${ }^{16}$ It resembles the position of Yablo (2005), although within a conceptualist framework apparently foreign to Yablo's own assumptions.

${ }^{17}$ Hence I here defend a hermeneutic fictionalism: the best interpretation of mathematical language is as uncommitted to mathematical objects.

\section{References}

Boolos, G.: 1971, 'The Iterative Conception of Set'. Journal of Philosophy 68, 215-32.

Chalmers, D.: 1996, The Conscious Mind. New York: Oxford University Press.

Feigenson, L., S. Carey, and E. Spelke: 2002, 'Infant's Discrimination of Number vs. Continuous Extent'. Cognitive Psychology 44, 33-66.

Field, H.: 1980, Science Without Numbers. Princeton, NJ: Princeton University Press.

Frege, G.: 1884, The Foundations of Arithmetic. Oxford: Blackwell. Translated by J. L. Austin.

Gödel, K.: 1964, 'What is Cantor's Continuum Problem?'. In: P. Benacerraf and H. Putnam (eds.): Philosophy of Mathematics: Selected Readings. Cambridge: Cambridge University Press, pp. 470-85.

Jackson, F.: 1998, From Metaphysics to Ethics: A Defence of Conceptual Analysis. Oxford: Oxford University Press.

Kitcher, P.: 1978, 'The Plight of the Platonist'. Noûs 12, 119-36.

Kitcher, P.: 1980, 'A Priori Knowledge'. Philosophical Review 89, 3-23.

Kripke, S.: 1980, Naming and Necessity. Cambridge, MA: Harvard University Press.

Lakatos, I.: 1976, Proofs and Refutations. Cambridge: Cambridge University Press.

Lewis, D.: 1970, 'How to Define Theoretical Terms'. Journal of Philosophy 67, 427-46.

Lewis, D.: 1973, Counterfactuals. Oxford: Blackwell.

Maddy, P.: 1997, Naturalism in Mathematics. Oxford: Oxford University Press.

McGee, V.: 1997, 'How We Learn Mathematical Language'. Philosophical Review 106, 3568.

Nolan, D.: 1996, 'The Credo of the Canberra Planners'. http://www.st-andrews .ac.uk/ dpn/docs/credo.htm.

Nolan, D.: forthcoming, 'Platitudes and Analysis'. In: D. Braddon-Mitchell and R. Nola (eds.): Naturalism and Analysis. Cambridge, MA: MIT Press.

O'Leary-Hawthorne, J. and H. Price: 1996, 'How to Stand Up for Non-Cognitivists'. Australasian Journal of Philosophy 74, 275-92.

Parsons, C.: 1995, 'Platonism and Mathematical Intuition in Kurt Gödel's Thought'. Bulletin of Symbolic Logic 1, 44-74.

Rosen, G.: 1990, 'Modal Fictionalism'. Mind 99, 327-54.

Smith, M.: 1994, The Moral Problem. Oxford: Blackwell. 
Soames, S.: 2004, Reference and Description. Princeton: Princeton University Press.

Stalnaker, R. C.: 1978, 'Assertion'. In: Context and Content. Oxford: Oxford University Press, pp. $78-95$.

Stalnaker, R. C.: 1996, 'Varieties of Supervenience'. Philosophical Perspectives 10, 221-42. van Fraassen, B. C.: 1989, Laws and Symmetry. Oxford: Oxford University Press.

Williamson, T.: 2006, 'Conceptual Truth'. Proceedings of the Aristotelian Society, Supplementary Volume 80.

Yablo, S.: 2001, 'Go Figure: A Path Through Fictionalism'. Midwest Studies in Philosophy 25, 72-102.

Yablo, S.: 2005, 'The Myth of the Seven'. In: M. E. Kalderon (ed.): Fictionalism in Metaphysics. Oxford: Oxford University Press, pp. 88-115.

Address for Offprints: Exeter College, Oxford, OX1 3DP, United Kingdom 